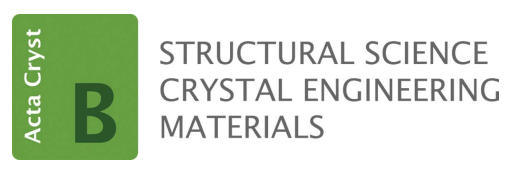

ISSN 2052-5206

Received 9 November 2015

Accepted 16 June 2016

Edited by P. Bordet, Institut Néel, France

Keywords: high- $T_{\mathrm{C}}$ superconductors; singlecrystal X-ray diffraction; chemical doping; intrinsic Josephson junctions.

CCDC references: $1486062 ; 1486063$

Supporting information: this article has supporting information at journals.iucr.org/b

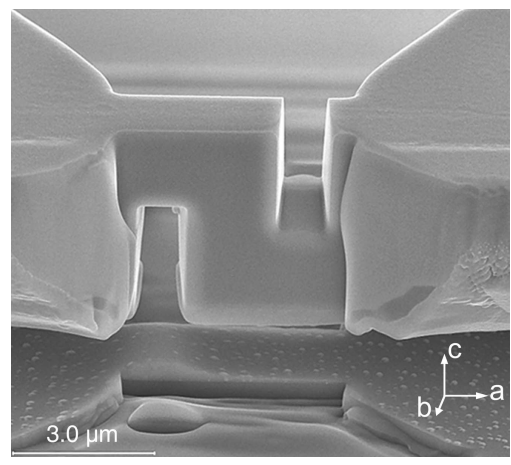

(C) 2016 International Union of Crystallography

\section{Effect of Al and Ca co-doping, in the presence of Te, in superconducting YBCO whiskers growth}

\author{
Lise Pascale, ${ }^{a}$ Marco Truccato, ${ }^{\mathrm{b}, \mathrm{c}}$ Lorenza Operti $^{\mathrm{a}, \mathrm{c}}$ and Angelo Agostino ${ }^{\mathrm{a}, \mathrm{c} *}$ \\ ${ }^{a}$ Department of Chemistry and CrisDi Interdepartmental Center for Crystallography, University of Torino, via P. Giuria 7, \\ Torino I-10125, Italy, ${ }^{\mathbf{b}}$ Department of Physics, University of Torino, via P. Giuria 1, Torino I-10125, Italy, and ${ }^{\mathbf{C}}$ NIS Centre \\ of Excellence, University of Torino, Italy. *Correspondence e-mail: angelo.agostino@unito.it
}

High- $T_{\mathrm{c}}$ superconducting cuprates (HTSC) such as $\mathrm{YBa}_{2} \mathrm{Cu}_{3} \mathrm{O}_{7-x}(\mathrm{YBCO})$ are promising candidates for solid-state $\mathrm{THz}$ applications based on stacks of intrinsic Josephson junctions (IJJs) with atomic thickness. In view of future exploitation of IJJs, high-quality superconducting YBCO tape-like single crystals (whiskers) have been synthesized from $\mathrm{Ca}-\mathrm{Al}$-doped precursors in the presence of Te. The main aim of this paper is to determine the importance of the simultaneous use of $\mathrm{Al}, \mathrm{Te}$ and $\mathrm{Ca}$ in promoting $\mathrm{YBCO}$ whiskers growth with good superconducting properties $\left(T_{\mathrm{c}}=79-84 \mathrm{~K}\right)$. Further, single-crystal Xray diffraction (SC-XRD) refinements of tetragonal YBCO whiskers $(P 4 / \mathrm{mmm})$ are reported to fill the literature lack of YBCO structure investigations. All the as-grown whiskers have also been investigated by means of $\mathrm{X}$-ray powder diffraction (XRPD), scanning electron microscopy (SEM) and energy dispersive $\mathrm{X}$-ray spectroscopy (EDS). Our results demonstrate that the interplay of $\mathrm{Ca}, \mathrm{Te}$ and $\mathrm{Al}$ elements is clearly necessary in order to obtain superconducting $\mathrm{YBCO}$ whiskers. The data obtained from SC-XRD analyses confirm the highly crystalline nature of the whiskers grown. $\mathrm{Ca}$ and $\mathrm{Al}$ enter the structure by replacing the $\mathrm{Y}$ and the octahedral coordinated Cu1 site, respectively, as in other similar orthorhombic compounds, while Te does not enter the structure of whiskers but its presence in the precursor is essential to the growth of the crystals.

\section{Introduction}

Stacks of intrinsic Josephson junctions (IJJs) with atomic thickness are naturally present in layered high- $T_{\mathrm{c}}$ superconducting cuprates (HTSCs), such as $\mathrm{Bi}_{2} \mathrm{Sr}_{2} \mathrm{CaCu}_{2} \mathrm{O}_{8+x}$ (Bi2212), $\mathrm{La}_{2}{ }_{x} \mathrm{Sr}_{x} \mathrm{CuO}_{4}$ (LSCO) and RE-123 (RE = Y, Eu, Gd, Dy, $\mathrm{Ho}, \mathrm{Er}, \mathrm{Tm}$ and $\mathrm{Lu}$ ), as a result of their crystal structure (Kawae et al., 2005; Kleiner et al., 1992; Kubo et al., 2008; Okutsu et al., 2008). IJJs can be employed as modular elements in the realization of several cryogenic devices such as $\mathrm{THz}$ sensors in Wang et al. (2001) and emitters in Ozyuzer et al. (2007), micro-SQUIDs in Sandberg \& Krasnov (2005) and quantum computers based on macroscopic quantum tunneling phenomena (Inomata et al., 2005; Martinis et al., 2005). Among the possible IJJ applications, high-frequency devices can take advantage of the large Josephson plasma frequency found in some HTSCs. In particular, $\mathrm{YBa}_{2} \mathrm{Cu}_{3} \mathrm{O}_{7-x}$ (Y-123) has the highest Josephson plasma frequency, close to a few $\mathrm{THz}$, because of its low anisotropy and high critical current density (Shibata \& Yamada, 1997), which makes it a suitable candidate for the fabrication of these kinds of devices. Furthermore, such properties could be modulated, for instance, by chemical substitutions, as already noticed for $\mathrm{Pb}$-doped $\mathrm{Bi}-2212$ in Kambara et al. (2011). Within this context, we recently investigated the effect of different cationic substitutions 
(Bertolotti et al., 2014) of anionic doping (Rahman Khan et al., 2009) and of X-ray nanobeam irradiation (Cagliero et al., 2009; Pagliero et al., 2014) on the electrical and structural properties of HTSC single crystals with high aspect ratios, also known as whiskers, belonging to $\mathrm{Y}-123$ and $\mathrm{Bi}-2212$ systems.

In view of the future exploitation of IJJs, whiskers are suitable structures for the study and the design of $\mathrm{THz}$ devices based on these junctions, which require a high homogeneity of properties on the micrometric scale. From this point of view, whiskers highly crystalline in nature, with excellent superconducting properties and low defect concentration represent crucial features for prototype fabrication. Moreover, starting from a micrometric cross section area, they are easily scalable by etching techniques down to submicrometric sizes and are also very suitable for three-dimensional machining, resulting in solid-state devices with a high degree of miniaturization (Inomata et al., 2002; Kawae et al., 2005; Okutsu et al., 2008; Pavlenko et al., 2009).

The growth of large amounts of $\mathrm{Bi}-2212$ whiskers was reported soon after the discovery of HTSC in Matsubara et al. (1989) and has been extensively studied in Badica et al. (2006). On the other hand, with regard to Y-123, whiskers were first obtained much later only by making simultaneous use of both Te and Ca (Nagao et al., 2003, 2004) in the precursor powders. Other growth strategies were investigated, for instance adding either $\mathrm{Sb}$ or Te as a single doping heteroelement (Nagao et al., 2005, 2010), but with worse results. Ca-doping of Y-123 increases the carrier density of underdoped material (Awana et al., 1994) as well as enhances both the values and the isotropy of the critical current density (Hammerl et al., 2000; Rutter et al., 2005), making Ca-doping essential for the YBCO whiskers growth. However, in just a few cases crystals have shown superconducting behavior and their yield always remained very limited. In our experiments, improving the work of Nagao et al. $(2003,2004,2005,2010)$, we have shown that the addition of limited amounts of $\mathrm{Al}_{2} \mathrm{O}_{3}$ powder to $\mathrm{Ca}$ doped precursors, in the presence of $\mathrm{Te}$, remarkably increases the amount of grown Y-123 whiskers. Therefore, we synthesized $\mathrm{Al}$-doped $\mathrm{Y}(\mathrm{Ca}) \mathrm{Ba}_{2} \mathrm{Cu}_{3} \mathrm{O}_{7-x}$ whiskers via a solid-state reaction method (Calore et al., 2013).

To date, the influence of Ca-doping in Y-123 has been widely studied from the point of view of its crystal structure, both for polycrystalline material and for single crystals not grown with the whisker technique (Böttger et al., 1997; Chen et al., 2000; Hijar et al., 1995). In principle, Ca should preferably substitute $\mathrm{Y}$ since the ionic radius of $\mathrm{Ca}^{2+}(r=1.12 \AA)$ is much closer to that of $\mathrm{Y}^{3+}(r=1.019 \AA)$ than to that of $\mathrm{Ba}^{2+}(r=$ $1.42 \AA$ ), and $\mathrm{X}$-ray structure refinements of single crystals have shown that at low Ca concentrations $(<11 \%) \mathrm{Y}^{3+}$ ions are actually substituted while, at higher concentrations $\mathrm{Ca}^{2+}$ also replaces $\mathrm{Ba}^{2+}$ (Böttger et al., 1997).

Concerning the influence of $\mathrm{Al}$ doping on the structure and superconducting properties of YBCO, this topic has been studied by many authors both in polycrystalline samples, in bulk single crystals and in whiskers. For instance, this element was either incorporated from alumina crucibles used during the synthesis process or added in the form of $\mathrm{Al}_{2} \mathrm{O}_{3}$ nano- particles to powders precursors, showing a substantial effect on the structure as well as the worsening of the transition temperature in both cases (Antal et al., 2010; Franck et al., 1987; Siegrist et al., 1987).

To the best of our knowledge, no study has been carried out on the importance of the simultaneous presence of $\mathrm{Al}$, Te and $\mathrm{Ca}$ in the precursors in promoting Y-123 whiskers growth with superconducting properties. Therefore, the present work is intended to underline the correlation of three such elements in the growth of YBCO whiskers by performing a comparison between $\mathrm{Al}$-doped, simultaneously $\mathrm{Ca}$ - and $\mathrm{Al}$-doped, $\mathrm{Te}$ - and Al-doped, and Ca-, Te- and Al-doped structures. Actually, it seems that tellurium oxide, in analogy with both aluminium and antimony oxides used as precursors, changes the phase diagram by creating mixed oxides, like $(\mathrm{BaCa})_{3} \mathrm{TeO}_{6}$. This change in the phase diagram promotes the creation of a melting zone named 'microcrucible' according to the most accredited theories (Badica et al., 2006), where the whiskers growth starts. Our experiments and related literature show how $\mathrm{Sb}$ and $\mathrm{Te}$ can increase the yield of whiskers as to $\mathrm{Al}$ (Nagao et al., 2003, 2010; Calore et al., 2013), without entering into the structure. While the superconducting properties of Y123 whiskers have already been investigated to a good extent, the structural study has been inexplicably overlooked so far so that only a single-crystal X-ray diffraction (SC-XRD) refinement can be found in the literature reporting an orthorhombic YBCO structure (Bertolotti et al., 2014). To fill this gap, in this paper we report SC-XRD refinements of tetragonal Y-123 whiskers. Moreover, all the as-grown crystals have been investigated by means of X-ray powder diffraction (XRPD), scanning electron microscopy (SEM) and energy dispersive $X$ ray spectroscopy (EDS). The superconducting properties have been evaluated and the resistivity measurements (R-T) are available as supporting information.

\section{Experimental}

\subsection{Whiskers syntheses}

In this work, doped single crystals (whiskers) of $\mathrm{YBa}_{2} \mathrm{Cu}_{3} \mathrm{O}_{7-x}$ were grown according to a flux method. Through this process, high-quality crystals grow as a consequence of free nucleation from a high-temperature solid solution, where the flux is a mixture of oxides belonging to the $\mathrm{Y}-\mathrm{Ba}-\mathrm{Cu}-\mathrm{O}$ phase diagram (Schneemeyer et al., 1987; Sun et al., 1990). Four different series of precursor powders were prepared by solid-state reaction from high-purity commercial $\mathrm{Y}_{2} \mathrm{O}_{3}$ (99.999\%), $\mathrm{BaCO}_{3}$ (99.9\%), $\mathrm{CuO}$ (99.99\%), $\mathrm{CaCO}_{3}$ (99.95\%), $\mathrm{TeO}_{2}(99.995 \%)$ and $\mathrm{Al}_{2} \mathrm{O}_{3}(99.998 \%$ ) (SigmaAldrich, Germany; Nagao et al., 2003). Each precursor had a nominal cationic ratio of Y:Ba:Cu:Ca:Te:Al = 1:2:3:(0.01.0):0.5:(0.025-0.05) or 1:2:3:(0.0-1.0):0:0.05. Powders were thoroughly mixed and calcined for $3 \mathrm{~h}$ at $1073 \mathrm{~K}$ in air and in $\alpha-\mathrm{Al}_{2} \mathrm{O}_{3}$ crucibles, with three intermediate grindings. Then the calcined powders were pressed into pellets of about $13 \mathrm{~mm}$ in diameter and $2 \mathrm{~mm}$ in thickness, which were subsequently put in a pure $\alpha-\mathrm{Al}_{2} \mathrm{O}_{3}$ boat and placed in a tube furnace for the 
Table 1

Synthesis identifier, nominal composition of precursors $\mathrm{YBa}_{2} \mathrm{Cu}_{3} \mathrm{Ca}_{u} \mathrm{Te}_{v} \mathrm{Al}_{\mathrm{w}} \mathrm{O}_{7-x}(\mathrm{Ca}=u, \mathrm{Te}=v$ and $\mathrm{Al}=w)$, heat treatment conditions ( $T_{\max }$ and $T_{\text {end }}$ ) and whisker results from different syntheses.

\begin{tabular}{lllllllll}
\hline & $\alpha 1$ & $\alpha 2$ & $\beta 1$ & $\beta 2$ & $\gamma 1$ & $\gamma 2$ & $\gamma 3$ & $\delta 1$ \\
\hline $\mathrm{Ca}=u$ & 1 & 0.5 & 0 & 0 & 0.25 & 0.25 & 1 & 0 \\
$\mathrm{Te}=v$ & 0.5 & 0.5 & 0.5 & 0.5 & 0.5 & 0.5 & 0 & 0 \\
$\mathrm{Al}=w$ & 0.05 & 0.05 & 0.05 & 0.025 & 0.05 & 0.025 & 0.05 & 0.05 \\
$T_{\max }(\mathrm{K})$ & 1278 & 1253 & 1278 & 1278 & 1253 & 1278 & 1278 & 1278 \\
$T_{\text {end }}(\mathrm{K})$ & 1178 & 1198 & 1198 & 1198 & 1198 & 1178 & 1198 & 1198 \\
Whiskers & $\mathrm{Y}-123$ & $\mathrm{Y}-123$ & $\mathrm{CuO}$ & $\mathrm{CuO}$ & None & None & None & None \\
\hline
\end{tabular}

thermal treatment. The whisker growth was carried out by heating the pellets in a controlled oxygen flow of $0.1 \mathrm{~L} \mathrm{~min}^{-1}$ under a thermal cycle of $T_{\max }=1253-1278 \mathrm{~K}, t_{\mathrm{dwell}}=5 \mathrm{~h}$ and $T_{\text {end }}=1178-1198 \mathrm{~K}$, where $T_{\max }$ is the maximum temperature of the heating ramp, $t_{\mathrm{dwell}}$ is the dwell time at $T_{\max }$ and $T_{\text {end }}$ is the end-point temperature of the cooling ramp after which the oven was turned off for furnace cooling. Differential thermal analysis (DTA) and power diffraction determinations were made on each series of precursors to define the best thermal conditions. All the syntheses were performed following the constant heating and cooling rates of $5 \mathrm{~K} \mathrm{~min}^{-1}$ and $1 \mathrm{~K} \mathrm{~h}^{-1}$, respectively.

\subsection{SEM/EDS analysis}

In order to evaluate the surface morphology and the cationic stoichiometry of synthesized whiskers, some electron micrographs and energy dispersive spectrometer (EDS) measurements were performed by means of a Cambridge S-360 scanning electron microscope (SEM) equipped with an Oxford Inca Energy 200 EDS system and the INCA Oxford software.

\subsection{Single-crystal X-ray diffraction}

All crystal structures were investigated by single-crystal Xray diffraction (SC-XRD). Intensities were collected at $293 \mathrm{~K}$ on an Xcalibur, Ruby, Gemini R Ultra diffractometer (Agilent Technologies UK Ltd), operating at $50 \mathrm{kV}$ and $40 \mathrm{~mA}$ with graphite-monochromated Mo $K \alpha$ radiation, by using an $\omega$ scan technique $\left(\Delta \omega=1.0^{\circ}\right)$. CrysAlisPro software has been used for data collection and reduction (peak intensities integration, background evaluation, cell dimensions and absorption correction; Agilent, 2012). Space-group determination, structure solution with direct methods (heavy-atom method) and refinement on $F^{2}$ have been performed by means of JANA2006 software (Petricek et al., 2014). The refinements were done using harmonic atomic displacement parameters for all atoms, except $\mathrm{O} 2$ and $\mathrm{O} 3$ sites. The occupancy factors (OFs) of all crystallographic sites were refined taking in account all the possible atomic combinations. Only the proven substitution was reported. A numerical absorption correction was applied by a Gaussian integration method over the crystal shape.

\subsection{Powder X-ray diffraction}

Powder X-ray diffraction (XRPD) data were obtained by means of an X'Pert Panalytical powder diffractometer, equipped with a $\mathrm{Cu}$ target and an $\mathrm{X}^{\prime}$ Celerator ultrafast line detector. The diffraction intensities were collected in a $\theta-\theta$
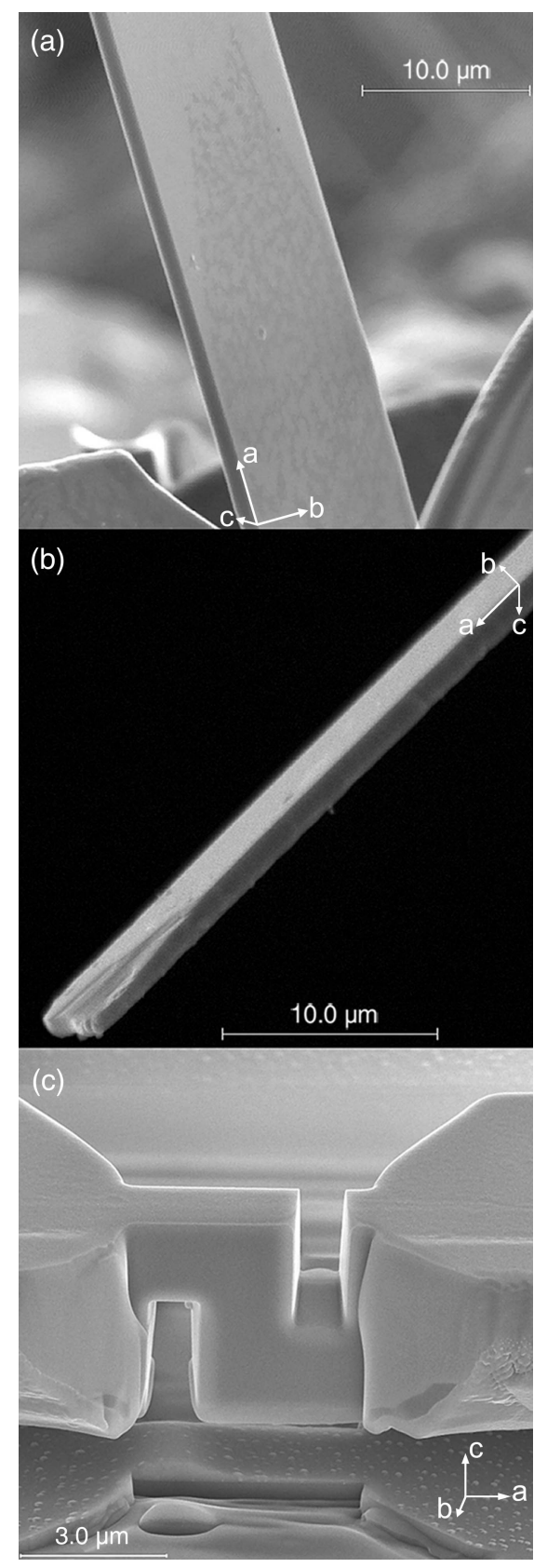

Figure 1

Scanning electron microscope (SEM) images of most common mediumsized Al-doped (Y,Ca)-123 whiskers obtained from batch $(a) \alpha 1$ and $(b)$ $\alpha 2$. The surface structure is very regular whatever the nominal composition of the precursor. (c) An SEM picture of the YBCO microdevice investigated in our electrical study. This whisker, selected from batch $\alpha 1$, has undergone different steps of micro-machining by means of a focused ion beam (FIB) instrument in order to fabricate stacks of intrinsic Josephson junctions. First, its cross-sectional area has been progressively decreased, then two trenches have been etched to force the current along the $c$-axis of the chip. 
Table 2

Crystal data for single crystals $\beta 1$ and $\beta 2$, and for phase $\delta 1$.

\begin{tabular}{lllll}
\hline & $\beta 1$ & $\beta 2$ & $\delta 1(\mathrm{Y}-123)$ & $\delta 1(\mathrm{Y}-211)$ \\
\hline Crystal system & Monoclinic & Monoclinic & Orthorhombic & Orthorhombic \\
Space group & $C 12 / c 1$ & $C 12 / c 1$ & $P m m m$ & $P n m a$ \\
$a, b, c(\AA)$ & $4.690(1), 3.420(1), 5.131(1)$ & $4.653(3), 3.410(1), 5.108(2)$ & $3.8303(5), 3.8803(5), 11.6669(13)$ & $5.6590(1), 7.1325(1), 12.1792(2)$ \\
$\beta\left(^{\circ}\right)$ & $99.54(2)$ & $99.48(1)$ & 90 & 90 \\
\hline
\end{tabular}

geometry, with $\mathrm{Cu} K \alpha$ radiation and an angular step size of $0.002^{\circ}$. Data analyses were performed using a Le Bail approach by means of the $J A N A 2006$ software.

\section{Results and discussion}

Table 1 summarizes the synthesis identifiers, the nominal composition of different precursors $\mathrm{YBa}_{2} \mathrm{Cu}_{3} \mathrm{Ca}_{u} \mathrm{Te}_{v} \mathrm{Al}_{w} \mathrm{O}_{7-x}$ employed, the experimental details ( $T_{\max }$ and $\left.T_{\text {end }}\right)$ and the kind of whisker obtained from the various syntheses. First, we have been able to obtain Y-123 single-crystal whiskers only starting from the precursors $\alpha 1$ and $\alpha 2$, and these crystals turned out to be superconductors with $T_{\mathrm{c}}$ values of 79.8 and $83.8 \mathrm{~K}$, respectively ( $R$ versus $T$ measurements are available as supporting information). SEM analyses were performed in order to obtain a morphological characterization.

Fig. 1 shows some SEM micrographs for the $\alpha 1$ (panel a) and $\alpha 2$ (panel b) synthesis batches. Our doped-YBCO whiskers are characterized by a quite regular tape-like structure of $\sim 0.2-1 \mathrm{~mm}$ in length, grown with a preferential direction and with a flat plane surface $10-35 \mu \mathrm{m}$ in width and $2-10 \mu \mathrm{m}$ in thickness. In order to obtain information about the elemental composition, EDS measurements were also performed. From a qualitative point of view, this analysis allows us to state that all whiskers grown from starting batches $\alpha 1$ and $\alpha 2$ contain Y, $\mathrm{Ba}, \mathrm{Cu}, \mathrm{O}, \mathrm{Ca}$ and $\mathrm{Al}$ but not Te. This outcome is in agreement with other similar compounds reported in Bertolotti et al. (2014) and Nagao et al. (2003). However, from a quantitative point of view, the evaluation of $\mathrm{Al}$ content could not be performed due to overlap of the corresponding peak with the Y $K \alpha$ one. The oxygen content could not be accurately determined either. Therefore, we could not define the corresponding stoichiometric compositions.

Data reported in Table 1 summarize the best conditions defined by DTA for each synthesis of YBCO after the variation of calcium in the stoichiometry. Some syntheses which failed significantly were also reported. During the experimental process, a decrease in nominal calcium content, i.e. from 1.0 in the $\alpha 1$ precursor to 0.5 in the $\alpha 2$ precursor, causes a reduction in the size of the obtained crystals. Probably the creation of intermediate oxide precursors, as $(\mathrm{BaCa})_{3} \mathrm{TeO}_{6}$, is crucial to increase the crystal size of the whiskers. In fact, reducing the amount of $\mathrm{Ca}$ in the pellet (see $\gamma 1$ and $\gamma 2$ syntheses) means that the growth of whiskers is no longer detected. However, while removing it completely (see precursors $\beta 1$ and $\beta 2$ ), an anomalous and concurrent growth of $\mathrm{CuO}$ whiskers occurs. This clearly indicates the necessity of introducing $\mathrm{Ca}$ in the precursors in order to obtain YBCO whiskers. Moreover, it is noticeable that the content in $\mathrm{Al}$, within the range of concentrations used in this paper, does not affect the type of whisker which we obtain, which can be Y-123 or $\mathrm{CuO}$, or neither. What we observe instead is that the content in Al strongly influences the yield from the synthesis. Indeed, according to the $\mathrm{Al}$ content in the precursor, what varies is the number of nucleation centers present on the pellet, as explained by the 'microcrucible' model in Badica et al. (2012) and Boston et al. (2014), and therefore the final number of whiskers grown. With regard to Te, its presence in the precursor seems to be essential to the growth of whisker crystals since, when removed, we do not obtain any whisker and the resulting sintered precursor pellet shows the presence of YBCO polycrystalline material (see $\gamma 1, \gamma 2$ and $\gamma 3$ ) or a mixed Y-123/Y-211 phase (see $\delta 1$ ).

The $\mathrm{CuO}$ whiskers $\beta 1$ and $\beta 2$ were identified by means of SC-XRD, while the Y-123/Y-211 phase $\delta 1$ was assigned by XRPD data. The crystal systems, space groups and lattice parameters of crystals $\beta 1, \beta 2$ and phase $\delta 1$ are reported in Table 2.

Crystals $\alpha 1$ and $\alpha 2$ were structurally studied by SC-XRD. The experimental details of the refinements are given in Table 3. Atomic coordinates, equivalent isotropic displacement parameters obtained from single-crystal refinements and the list of interatomic distances are available as supporting information. Both crystals studied are characterized by a tetragonal non-stoichiometric stacked perovskite-rock saltperovskite structure with space group $P 4 / \mathrm{mmm}(Z=1)$, by similar lattice parameters and by similar atomic coordinations, but differ in the composition (notably $\mathrm{Ca}$ and $\mathrm{Al}$ ) as well as in the doping content $(\mathrm{O})$. Structure refinements converged at $R \simeq 0.020$ and $0.027<w R<0.038$.

\subsection{Crystal $a 1$}

Fig. 2 shows the tetragonal structure of crystal $\alpha 1$. Y and $\mathrm{Ba}$ ions occupy the $1 \mathrm{~d}(4 / \mathrm{mmm})$ and the $2 \mathrm{~h}(4 \mathrm{~mm})$ Wyckoff sites, respectively. $\mathrm{Cu} 1$, which is in the $1 a(4 / \mathrm{mmm})$ Wyckoff site, achieves an octahedral coordination geometry where four $\mathrm{O} 3$ ions ( $2 f$ Wyckoff site, $m m m$ ) form the plane and two O1 (1b Wyckoff site, $4 / \mathrm{mmm}$ ) form the vertices. Finally, the $\mathrm{Cu} 2$ ion occupies the $2 \mathrm{~g}$ Wyckoff site $(4 \mathrm{~mm})$ and has a slightly distorted square-pyramidal coordination, with four $\mathrm{O} 2$ ions $(4 i$ Wyckoff site, $2 \mathrm{~mm}$ ) in the plane and a weak interaction with apical O1.

Crystal $\alpha 1$ is Ca- and Al-doped but Te-free. This result is in agreement with our EDS analysis and ICP-MS (ion-coupled plasma mass spectrometry) analysis previously reported in 
Table 3

Experimental details: crystal data, data collection and structure refinements for the single crystals $\alpha 1$ and $\alpha 2$.

For all structures: $Z=1$ and refinement was with 0 restraints. Experiments were carried out at $293 \mathrm{~K}$ with Mo $K \alpha$ radiation using an Xcalibur, Ruby, Gemini ultra diffractometer by using an $\omega$-scan technique. Refinement was on 20 parameters.

\begin{tabular}{lll}
\hline & Crystal $\alpha 1$ & Crystal $\alpha 2$ \\
\hline Crystal data & & \\
Chemical formula & $\mathrm{Y}_{0.95} \mathrm{Ca}_{0.05} \mathrm{Ba}_{2} \mathrm{Cu}_{2.84} \mathrm{Al}_{0.16} \mathrm{O}_{6.75}$ & $\mathrm{Y}_{0.92} \mathrm{Ca}_{0.08} \mathrm{Ba}_{2} \mathrm{Cu}_{2.90} \mathrm{Al}_{0.10} \mathrm{O}_{6.89}$ \\
$M_{\mathrm{r}}$ & 654.01 & 657.02 \\
Crystal system, space group & Tetragonal, $P 4 / \mathrm{mmm}$ & Tetragonal, $P 4 / m m m$ \\
$a, c(\AA)$ & $3.8551(3), 11.6782(8)$ & $3.8526(5), 11.6660(15)$ \\
$V\left(\AA^{3}\right)$ & $173.56(3)$ & $173.15(5)$ \\
$F(000)$ & 288.5 & 290 \\
$D_{x}\left(\mathrm{Mg} \mathrm{m}^{-3}\right)$ & 6.257 & 6.301 \\
$\mu\left(\mathrm{mm}^{-1}\right)$ & 27.61 & 27.65 \\
Crystal size $(\mathrm{mm})$ & $0.29 \times 0.02 \times 0.01$ & $0.23 \times 0.02 \times 0.01$ \\
& & \\
Data collection & $\mathrm{Gaussian}$ integration & $\mathrm{Gaussian}$ integration \\
Absorption correction & $0.197,0.811$ & $0.127,0.821$ \\
$T_{\min }, T_{\max }$ & $3779,186,174$ & $1215,186,162$ \\
No. of measured, independent and & & \\
$\quad$ observed $[I>3 \sigma(I)]$ reflections & 0.038 & 0.033 \\
$R_{\text {int }}$ & 0.683 & 0.683 \\
$(\sin \theta / \lambda)_{\max }\left(\AA^{-1}\right)$ & & \\
Refinement & $0.021,0.038,1.52$ & $0.020,0.027,0.92$ \\
$R\left[F^{2}>2 \sigma\left(F^{2}\right)\right], w R\left(F^{2}\right), S$ & 174 & 162 \\
No. of reflections & $1.32,-1.45$ & $0.47,-0.52$ \\
$\Delta \rho_{\max }, \Delta \rho_{\min }\left(\mathrm{e} \AA^{-3}\right)$ & & \\
\hline
\end{tabular}

Computer programs: CrysAlisPro (Agilent, 2012), JANA2006, Version 28/04/2014 (Petricek et al., 2014). similar compounds (Bertolotti et al., 2014; Nagao et al., 2003), only the $\mathrm{Y}^{3+}$ site is substituted by $\mathrm{Ca}^{2+}$ with a molar ratio $\mathrm{Y}^{3+} / \mathrm{Ca}^{2+}$ of $0.95 / 0.05$. This crystal also contains $\mathrm{Al}$ ions; the refinement shows that only $\mathrm{Cu} 1$ is substituted and the molar ratio $\mathrm{Cu} 1 / \mathrm{Al}$ is $0.95 / 0.05$. The OFs of all $\mathrm{O}$ atoms have been refined: only $\mathrm{O} 3$ has shown that the site occupancy is not full $(\mathrm{OF}=0.38)$. The experimental formula of crystal $\alpha 1$ is therefore $\left(\mathrm{Y}_{0.95} \mathrm{Ca}_{0.05}\right) \mathrm{Ba}_{2.00}\left(\mathrm{Cu}_{2.84} \mathrm{Al}_{0.16}\right) \mathrm{O}_{6.75}$.

The changes engendered in Y-123 whisker structure by $\mathrm{Ca}$ doping need to be discussed. Since the structural parameters of YBCO depend on the doping level as well as on the oxygen content, we have taken, as a reference from the literature, the data of an undoped tetragonal single crystal with a similar oxygen content (Okamura et al., 1987). In this way, it is possible to separate the changes induced by $\mathrm{Ca}$ doping from those due to oxygen stoichiometry. The main effect of $\mathrm{Y}^{3+}$ $(r=1.019 \AA)$ substitution by the larger $\mathrm{Ca}^{2+}(r=1.12 \AA)$ is an increase in the distance between the $\mathrm{Cu} 2-\mathrm{O} 2$
Bertolotti et al. (2014). The refinement was carried out allowing $\mathrm{Ca}^{2+}$ to replace both $\mathrm{Y}^{3+}$ and $\mathrm{Ba}^{2+}$ and, as in other

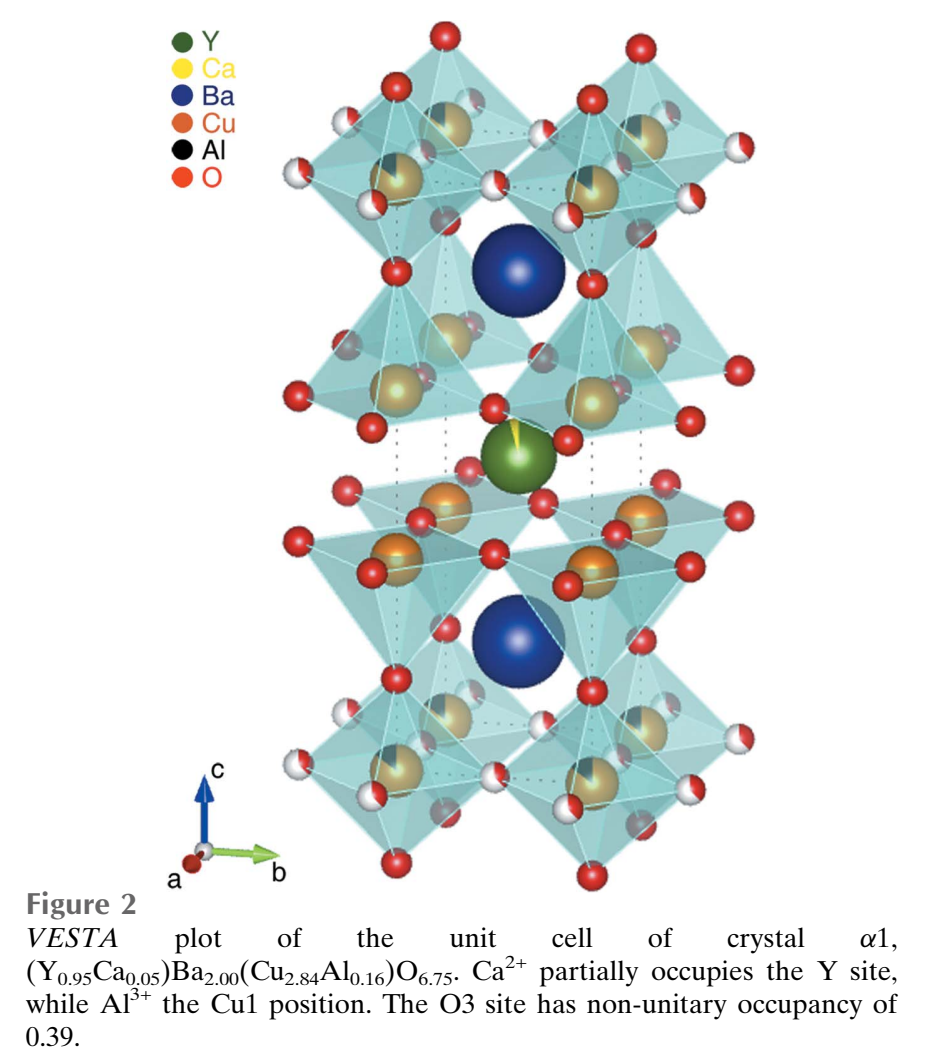

planes, separated by $\mathrm{Y}(\mathrm{Ca})$. Indeed, the atomic distance $\mathrm{Cu} 2-\mathrm{Cu} 2$ changes from 3.280 (7) $\AA$ in the undoped Y-123 to 3.3381 (5) $\AA$ in our $\alpha 1 \mathrm{Y}(\mathrm{Ca})-123$ whisker, taking $\mathrm{Cu} 2$ closer to the apical oxygen $\mathrm{O} 1$ [the distance $\mathrm{Cu} 2-\mathrm{O} 1$ is reduced from 2.529 (24) to 2.3512 (3) $\AA$ ]. This effect has already been observed in other $\mathrm{Y}-\mathrm{Ba}-\mathrm{Cu}-\mathrm{O}$ materials doped with $\mathrm{Ca}$, as single crystals of YBCO (Böttger et al., 1997), Y-247 and Y-124 (Schwer et al., 1994), as well as in polycrystalline Y-123 (Böttger et al., 1996).

\subsection{Crystal $\alpha 2$}

The structure of crystal $\alpha 2$ is very close to crystal $\alpha 1$, as described above. The refinement shows that $\mathrm{Ca}^{2+}$ partially replaces $\mathrm{Y}^{3+}$, while only the $\mathrm{Cu} 1$ ion is substituted by $\mathrm{Al}^{3+}$. In this case, the molar ratios $\mathrm{Y} / \mathrm{Ca}$ and $\mathrm{Cu} 1 / \mathrm{Al}$ are $0.92 / 0.08$ and $0.97 / 0.03$, respectively. Contrary to what we could expect from the nominal composition of precursors (see $\alpha 1$ and $\alpha 2$ in Table 1), Ca content in this crystal is higher with respect to what we found in crystal $\alpha 1$. However, Ca concentrations we used in both $\alpha 1$ and $\alpha 2$ pellets were, respectively, 4.6 and 9 times greater than the maximum $\mathrm{Y} / \mathrm{Ca}$ replacement level found in single crystals of Ca-doped YBCO (Calore et al., 2013). Furthermore, from both $\alpha 1$ and $\alpha 2$ single-crystal structure refinements, Ca was found to occupy only the $\mathrm{Y}$ site at low levels of doping (less than $11 \%$ ). The occupancy of the $\mathrm{O} 3$ site has been refined to a value of 0.44 , resulting in an experi- 
mental formula for crystal $\alpha 2$ of $\left(\mathrm{Y}_{0.92} \mathrm{Ca}_{0.08}\right) \mathrm{Ba}_{2.00^{-}}$ $\left(\mathrm{Cu}_{2.90} \mathrm{Al}_{0.10}\right) \mathrm{O}_{6.89}$.

\section{Conclusions}

High-quality superconducting YBCO-doped tape-like single crystals were grown from simultaneously $\mathrm{Ca}$-Al-doped precursors, in the presence of Te. We observed that an increment of the Ca content in the structure causes a reduction of the length of the crystals. The addition of alumina in the precursor pellet strongly influences the yield of the synthesis but, at any content, the $\mathrm{Al}$ incorporation in the structure is nearly constant and occurs only at the $\mathrm{Cu} 1$ site. Finally, Te does not enter into the structure of whiskers but its presence in the precursor is essential to the growth of crystals (see the syntheses of $\gamma 1, \gamma 2, \gamma 3$ and $\delta 1$ ). Summarizing together these experimental results with the reported literature shows that the interplay of these three doping elements is clearly necessary in order to obtain Y-123 whiskers. Additional investigation is required to better understand the growth mechanism and shape formation of these singular micro-crystals.

The data obtained from SC-XRD analyses on as-grown samples confirm the highly crystalline nature of whiskers. All the examined crystals are characterized by a single tetragonal domain $(P 4 / \mathrm{mmm})$. Ca and $\mathrm{Al}$ enter into the structure by replacing the $\mathrm{Y}$ and the octahedral coordinated $\mathrm{Cu} 1$ site, respectively, as in other similar orthorhombic compounds.

At present, further studies on the superconducting properties of these micrometric tetragonal doped-YBCO whiskers are in progress. The preliminary results are encouraging and offer new perspectives for future work on chemically induced IJJ modulation for the generation of materials with new functionalities.

\section{References}

Agilent (2012). CrysAlisPro, Version 1.171.36.28. Agilent Technologies UK Ltd, Oxford, England.

Antal, V., Zmorayová, K., Kováč, J., Kavečanský, V., Diko, P., Eisterer, M. \& Weber, H. W. (2010). Supercond. Sci. Technol. 23, 065014.

Awana, V. P. S., Tulapurkar, A., Malik, S. K. \& Narlikar, A. V. (1994). Phys. Rev. B, 50, 594-596.

Badica, P., Agostino, A., Khan, M. M. R., Cagliero, S., Plapcianu, C., Pastero, L., Truccato, M., Hayasaka, Y. \& Jakob, G. (2012). Supercond. Sci. Technol. 25, 105003.

Badica, P., Togano, K., Awaji, S., Watanabe, K. \& Kumakura, H. (2006). Supercond. Sci. Technol. 19, R81-R99.

Bertolotti, F., Calore, L., Gervasio, G., Agostino, A., Truccato, M. \& Operti, L. (2014). Acta Cryst. B70, 236-242.

Boston, R., Schnepp, Z., Nemoto, Y., Sakka, Y. \& Hall, S. R. (2014). Science, 344, 623-626.

Böttger, G., Mangelschots, I., Kaldis, E., Fischer, P., Krüger, C. \& Fauth, F. (1996). J. Phys. Condens. Matter, 8, 8889-8905.

Böttger, G., Schwer, H., Kaldis, E. \& Bente, K. (1997). Physica C, 275 , 198-204.

Cagliero, S., Piovano, A., Lamberti, C., Rahman Khan, M. M., Agostino, A., Agostini, G., Gianolio, D., Mino, L., Sans, J. A., Manfredotti, Ch. \& Truccato, M. (2009). J. Synchrotron Rad. 16, 813-817.
Calore, L., Rahman Khan, M. M., Cagliero, S., Agostino, A., Truccato, M. \& Operti, L. (2013). J. Alloys Compd, 551, 19-23.

Chen, C., Wondre, F., Chowdhury, A. J. S., Hodby, J. W. \& Ryan, J. F. (2000). Physica C, 341-348, 589-592.

Franck, J. P., Jung, J. \& Mohamed, M. A. K. (1987). Phys. Rev. B, 36, 2308-2310.

Hammerl, G., Schmehl, A., Schulz, R. R., Goetz, B., Bielefeldt, H., Schneider, C. W., Hilgenkamp, H. \& Mannhart, J. (2000). Nature, 407, 162-164.

Hijar, C. A., Stern, C. L., Poeppelmeier, K. R., Rogacki, K., Chen, Z. \& Dabrowski, B. (1995). Physica C, 252, 13-21.

Inomata, K., Kawae, T., Kim, S. J., Nakajima, K., Yamashita, T., Nagao, M. \& Maeda, H. (2002). Physica C, 372-376, 335-338.

Inomata, K., Sato, S., Nakajima, K., Tanaka, A., Takano, Y., Wang, H. B., Nagao, M., Hatano, H. \& Kawabata, S. (2005). Phys. Rev. Lett. 95, 107005.

Kambara, H., Kakeya, I. \& Suzuki, M. (2011). Physica C, 471, 754757.

Kawae, T., Nagao, M., Takano, Y., Wang, H. B., Hatano, T. \& Yamashita, T. (2005). Physica C, 426-431, 1479-1483.

Kleiner, R., Steinmeyer, F., Kunkel, G. \& Müller, P. (1992). Phys. Rev. Lett. 68, 2394-2397.

Kubo, Y., Tanaka, T., Takahide, Y., Ueda, S., Okutsu, T., Islam, A. T. M. N., Tanaka, I. \& Takano, Y. (2008). Physica C, 468, 1922 1924.

Martinis, J. M., Cooper, K. B., McDermott, R., Steffen, M., Ansmann, M., Osborn, K. D., Cicak, K., Oh, S., Pappas, D. P., Simmonds, R. W. \& Yu, C. C. (2005). Phys. Rev. Lett. 95, 210503-210506.

Matsubara, I., Kageyama, H., Tanigawa, H., Ogura, T., Yamashita, H. \& Kawai, T. (1989). Jpn. J. Appl. Phys. 28, L1121-L1124.

Nagao, M., Kawae, T., Yun, K., Wang, H., Takano, Y., Hatano, T., Yamashita, T., Tachiki, M., Maeda, H. \& Sato, M. (2005). J. Appl. Phys. 98, 073903.

Nagao, M., Sato, M., Maeda, H., Yun, K. S., Takano, Y., Hatano, T. \& Kim, S. (2003). Appl. Phys. Lett. 82, 1899-1901.

Nagao, M., Sato, M., Tachiki, Y., Miyagawa, K., Tanaka, M., Maeda, H., Yun, K. S., Takano, Y. \& Hatano, T. (2004). Jpn. J. Appl. Phys. 43, L324-L327.

Nagao, M., Watauchi, S., Tanaka, I., Okutsu, T., Takano, Y., Hatano, T. \& Maeda, H. (2010). Jpn. J. Appl. Phys. 49, 033101.

Okamura, F. P., Sueno, S., Nakai, I. \& Ono, A. (1987). Mater. Res. Bull. 22, 1081-1085.

Okutsu, T., Ueda, S., Ishii, S., Nagasawa, M. \& Takano, Y. (2008). Physica C, 468, 1929-1931.

Ozyuzer, L., Koshelev, A. E., Kurter, C., Gopalsami, N., Li, Q., Tachiki, M., Kadowaki, K., Yamamoto, T., Minami, H., Yamaguchi, H., Tachiki, T., Gray, K. E., Kwok, W. K. \& Welp, U. (2007). Science, 318, 1291-1293.

Pagliero, A., Mino, L., Borfecchia, E., Truccato, M., Agostino, A., Pascale, L., Enrico, E., De Leo, N., Lamberti, C. \& MartínezCriado, G. (2014). Nano Lett. 14, 1583-1589.

Pavlenko, V. N., Latyshev, Y. I., Chen, J., Gaifullin, M. B., Irzhak, A., Kim, S. J. \& Wu, P. H. (2009). JETP Lett. 89, 249-252.

Petricek, V., Dusek, M. \& Palatinus, L. (2014). Z. Kristallogr. 229, 345-352.

Rahman Khan, M. M., Cagliero, S., Agostino, A., Beagum, M., Plapcianu, C. \& Truccato, M. (2009). Supercond. Sci. Technol. 22, 085011.

Rutter, N. A., Durrell, J. H., Blamire, M. G., MacManus-Driscoll, J. L., Wang, H. \& Foltyn, S. R. (2005). Appl. Phys. Lett. 87, 162507.

Sandberg, M. \& Krasnov, V. M. (2005). Phys. Rev. B, 72, 212501.

Schneemeyer, L. F., Waszczak, J. V., Siegrist, T., van Dover, R. B., Rupp, L. W., Batlogg, B., Cava, R. J. \& Murphy, D. W. (1987). Nature, 328, 601-603.

Schwer, H., Kaldis, E., Karpinski, J. \& Rossel, C. (1994). J. Solid State Chem. 111, 96-103. 


\section{research papers}

Shibata, H. \& Yamada, T. (1997). Physica C, 293, 191-195.

Siegrist, T., Schneemeyer, L. F., Waszczak, J. V., Singh, N. P., Opila, R. L., Batlogg, B., Rupp, L. W. \& Murphy, D. W. (1987). Phys. Rev. $B, \mathbf{3 6}, 8365-8368$.
Sun, B. N., Hartman, P., Woensdregt, C. F. \& Schmid, H. (1990). J. Cryst. Growth, 100, 605-614.

Wang, H. B., Wu, P. H. \& Yamashita, T. (2001). Phys. Rev. Lett. 87, 107002-107005. 